\title{
Mode Propagation in X-Ray Waveguides
}

\author{
J. Choi*, J. Jung, and T. Kwon \\ Photonics Lab, Department of Physics, Dankook University Cheonan, 330-714, Republic of Korea
}

(Received February 21, 2008 : revised May 28, 2008 : accepted May 28, 2008)

\begin{abstract}
Single-mode propagation conditions of X-ray waveguides are investigated by numerical calculations in order to understand the importance of waveguide design parameters, such as core thickness and the optical constants of waveguide materials, on the transmission and coherence properties of the waveguide. The simulation code for mode analyzing is developed based on a numerical solution of the parabolic wave equation. The initial boundary value problem is solved numerically using a finite-difference scheme based on the Crank-Nicolson scheme. The E-field intensities in a core layer are calculated at an X-ray energy of $8.0 \mathrm{keV}$ for air and beryllium(Be) core waveguides with different cladding layers such as $\mathrm{Pt}, \mathrm{Au}, \mathrm{W}, \mathrm{Ni}$ and $\mathrm{Si}$ to determine the dependence on waveguide materials. The highest E-field intensity radiated at the exit of the waveguide is obtained from the Pt cladded beryllium core with a thickness of $20 \mathrm{~nm}$. However, the intensity from the air core waveguide with $\mathrm{Pt}$ cladding reaches $64 \%$ of the Be-Pt waveguide. The dependence on the core thickness, which is the major parameter used to generate a single mode in the waveguide, is investigated for the air-Pt, and Be-Pt waveguides at an X-ray energy of $8.0 \mathrm{keV}$. The mode profiles at the exit are shown for the single mode at a thickness of up to $20 \mathrm{~nm}$ for the air-Pt and the Be-Pt waveguides.
\end{abstract}

Keyword: X-ray waveguide, single mode, channel waveguide, Finite-difference method

OCIS codes : (340.0340) X-ray optics; (340.7480) X-rays; (310.2790) Guided waves; (230.7400) Waveguides, slab.

\section{INTRODUCTION}

There are increasing research efforts on the X-ray optical devices to produce small X-ray spots with a dimension of nanometers in the field of device fabrications and applications such as micro, nano-imaging and microdiffraction experiments. A micron-sized X-ray spot can be generated using conventional X-ray optic components that consist of a compound refractive lens, a capillary optic and a zone plate lens. The parabolic compound refractive lenses (CRL) provide micron- to nano-sized $\mathrm{X}$-ray spots $[1,2]$. Nanometer-sized focused beams have been produced by using a Fresnel zone plate within the soft X-ray spectrum range [3,4]. A spatial resolution better than $30 \mathrm{~nm}$ is reached using a zone plate $[5,6]$. But it is difficult to obtain a coherent nano-beam at an X-ray energy of $8 \mathrm{keV}$ and above because of strong absorption and phase shifts with increasing photon energy. However, recent theoretical and experimental

*Corresponding author: choi@dku.edu research results have shown that the linear zone plate can be used to focus hard X-rays up to $20 \mathrm{keV}$ [7-10]. A spot size of about $90 \mathrm{~nm}$ has been achieved with glass capillary optics [11]. But the capillary optics did not preserve the coherence of the X-ray beams. A pinhole can also be used to generate a micron-sized beam, but smaller, nanometer-sized coherent beams could not be obtained by pinholes. Even if, hypothetically, nano-sized pinholes were available, the output flux from the pinhole would not be enough for most applications. The first demonstration of sub-micrometer beam generation was conducted with a planar and a $2-\mathrm{D}$ waveguide [12]. A single mode X-ray waveguide was investigated for feasibility in terms of generating a smaller X-ray spot [13]. It provides X-ray spots of around $10 \mathrm{~nm}$, with high coherence [14].

Fully coherent X-ray beams are used to investigate the nanostructure of materials with the coherent diffraction imaging technique $[15,16]$. An X-ray waveguide way for X-ray optics to generate a fully coherent beam 
of X-rays for use as a source in the diffraction imaging process, because it produces a coherent and divergent $\mathrm{X}$-ray beam that is generated based on the principle of resonant beam coupling. The flux of X-rays at the exit of the waveguide is efficiently gained by the enhancement of the internal resonant field in the guiding layer [13]. An enhancement of the electric-field intensity takes place in a guiding layer with lower electronic density that is deposited between reflecting surfaces with higher density [17]. When the incident angle at the higher density is smaller than the critical angle $\alpha_{c}$, it is proportional to the square root of the phase drag coefficient $\sqrt{\delta}$. The incident beam penetrating into the layers with a lower electronic density than that of air is refracted at the interface and then totally reflected from the low-high electronic density interface. In this case, the X-ray standing wave is created at the lower electronic density layer, whose thickness $\mathrm{D}$ is given by [18]:

$$
D=\frac{\lambda}{2 \sin \alpha_{r}}
$$

where $\lambda$ is the wavelength of the incident $\mathrm{x}$-ray beam and $\alpha_{r}$ is the grazing incident angle at the total reflected interface. The incident X-ray beam can be directly coupled into the waveguide aperture, and the spatial acceptance depends on the cross-section of the core in the waveguide. Then, several modes can be excited simultaneously in the waveguide, depending on the thickness of the core. The waveguide parameter $V$ can be described by the refractive indices of the core and the cladding $n_{1}, n_{2}\left(n_{1}>n_{2}\right)$, respectively, and the thickness of the guiding layer [19].

$$
V=\sqrt{n_{1}-n_{2}} k d \approx \sqrt{2 \delta_{2}-2 \delta_{1}} k d
$$

The refractive index of the medium in the region of $\mathrm{X}$-rays is given by:

$$
n=1-\delta+i \beta
$$

The real part of the index of refraction for hard $\mathrm{X}$-rays is always slightly smaller than one. In order to utilize the X-ray waveguide, the core material must have a low electron density with a low absorption coefficient, and the cladding material has to have a high electron density with a sufficiently high potential wall for the formation of guided modes. To develop the Xray waveguide, it is necessary to understand the transmission and coherence properties of the waveguide depending on the waveguide design parameters, such as the core thickness and the optical constants of the materials.

In this paper, the mode transmission will be predicted by computer simulations for various waveguide materials and the core thickness of the X-ray waveguides. Then, the specifications of the X-ray waveguides that are fabricated from a beryllium (Be) core and an air core with a tungsten $(\mathrm{W})$ cladding are investigated for practical utilization of the X-ray waveguides.

\section{METHODS AND MATERIALS}

The computer simulations were carried out based on the numerical solution of the parabolic equation. The simulation code for mode analyzing is developed using a finite element method scheme based on CrankNicolson [20]. Consider the confined X-rays propagating along the $\mathrm{x}$-direction in a guiding core with thickness $d$ where the refractive index $n_{1}$ is larger than the refractive index $n_{2}$ in the cladding. The coordinate used in the numerical calculation is shown in Fig. 1. When Xray beams are incident on the front face of the waveguide, which is perpendicular to the direction of the wave propagation, then the horizontal component of the electric field is denoted by $\varphi$. The propagation of a monochromatic X-ray is described by the Helmholtz equation:

$$
\nabla^{2} \varphi+k^{2} n^{2}(x, y, z)=0
$$

where $k=\omega / c=2 \pi / \lambda$ is the wave number in free space.

In this study, one-dimensional confinement is considered i.e., the electric field and refractive index profiles are independent of $y$. The refractive indexes in the waveguide are:

$$
n=\left\{\begin{array}{cc}
n_{1} & 0<z<d \\
n_{2} & \text { else }
\end{array}\right.
$$

For the electric field inside the waveguide:

$$
\varphi(x, z)=\varphi(z) \exp (-i \beta x)
$$

where $\beta$ is called the propagation constant. Then, the Helmholtz equation can be reduced thus:

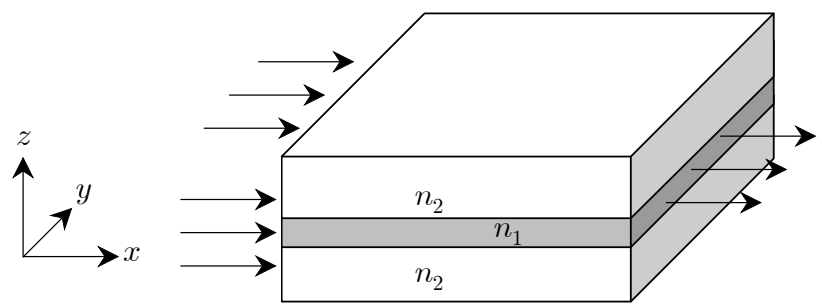

FIG. 1. A schematic of the X-ray waveguide coordinates for the numerical calculations 


$$
\frac{d^{2} \varphi(z)}{d t^{2}}+\left(n^{2} k^{2}-\beta^{2}\right) \varphi(z)=0
$$

The horizontal component of the electric field $\varphi(z)$ has to be continuous at the interface of the core and the cladding surfaces. Now, consider confined X-rays propagating along the $\mathrm{x}$-direction of the core with a thickness of $d$. When the beam of X-ray waves is incident on the entrance of the waveguide, one component propagates in the core and the other component travels in the cladding, which is quickly damped out. Thus, only the guided modes contribute to the generation of the electric field at the exit face of the waveguide. The wave equation for the one-dimensional confinement is independent of $\mathrm{y}$ and is given by:

$$
\left(\frac{\partial^{2}}{\partial x^{2}}+\frac{\partial^{2}}{\partial z^{2}}\right) \varphi+k^{2} n^{2}(x, z) \varphi=0
$$

Where the wave vector $\vec{k}$ is less than the critical angle of total reflection, $\varphi$ and oscillates very fast in the $\mathrm{x}$-axis. Thus, the numerical calculation requires a larger number of computing steps. In order to overcome this problem, $\varphi$ is written as the product of a function $u$ and $\exp (-i k x)$ which is given by:

$$
\varphi(x, y, z)=u(x, y, z) \exp (-i k x)
$$

where $u=u_{0} \exp (i k \delta x)$. So, the function $u$ oscillates much slower than $\varphi$.

Then the Helmholtz equation becomes:

$$
-2 i k \frac{\partial u}{\partial x}+\left(\frac{\partial^{2}}{\partial y^{2}}+\frac{\partial^{2}}{\partial z^{2}}\right) u+k^{2}(n-1) u=0
$$

This equation is known as the parabolic wave equation. For convenience we define:

$$
\begin{aligned}
& A=-\frac{i}{2 k}, \\
& F(x, y, z)=-\frac{i k}{2}\left[n^{2}(x, y, z)-1\right]
\end{aligned}
$$

Then Eq. (10) becomes:

$$
\frac{\partial u}{\partial x}=A\left(\frac{\partial^{2} u}{\partial^{2} y}+\frac{\partial^{2} u}{\partial^{2} z}\right)+F(x, y, z) u
$$

The parabolic wave equation in two dimensions can be used for a waveguide confined to one dimension by dropping any y-dependence:

$$
\frac{\partial u}{\partial x}=A\left(\frac{\partial^{2} u}{\partial^{2} z}\right)+F(x, z) u
$$

and the boundary conditions are given as:

$$
u(0, z)=u_{0}\left(0, z_{0}\right)
$$

$$
\begin{aligned}
& u\left(x, z_{0}\right)=u_{0}\left(x, z_{0}\right) \\
& u\left(x, z_{m}\right)=u_{0}\left(x, z_{m}\right)
\end{aligned}
$$

where $u_{0}(x, z)$ is an incident plane wave which is a damping plane wave in the cladding layer. The initial boundary value problem is solved numerically using a finite-difference scheme which is based on the CrankNicolson scheme, and has second-order accuracy of $\Delta x$ and $\Delta z$ [20]. The electromagnetic wave propagates away from the boundaries, so the partial derivatives in Eq. (13) become:

$$
\frac{u_{k}^{n+1}-u_{k}^{n}}{\Delta x}=\frac{A}{2}\left(\frac{u_{k-1}^{n}-2 u_{k}^{n}+u_{k+1}^{n}}{\Delta z^{2}}+\frac{u_{k-1}^{n+1}-2 u_{k}^{n-1}+u_{k+1}^{n+1}}{\Delta z^{2}}\right)+\frac{F_{k}^{n+\frac{1}{2}}}{2}\left(u_{k}^{n}+u_{k}^{n+1}\right)
$$

The source code of the numerical calculation is written with the IDL programming language and is based on Eq. (15). Each waveguide is confined to a finite number of guided modes depending on the core dimension, so that the number of modes propagating in a core can be found by $N=[V / \pi]_{\text {int }}$ where $\mathrm{V}$ is obtained from Eq. (2). The critical thickness has to be determined to fabricate an X-ray waveguide for single mode generation. When $\mathrm{X}$-rays are passing through an aperture, the critical width $W_{c}=\lambda / 2 \theta_{c}$ is dependent on the wavelength $\lambda$ and is weakly material dependent [14]. In particular, the critical width for the slab waveguide $d_{c}=\lambda / \sqrt{2 \delta_{2}-2 \delta_{1}}$ is defined using the parameters of the materials.

\section{RESULTS AND DISCUSSION}

Fig. 2 shows the calculated electric field intensities inside the core for different thicknesses at an X-ray energy of $8.0 \mathrm{keV}$. The waveguides consist of an air channel as a guiding layer with Pt cladding. The waveguide length has to be long enough to ensure sufficient absorption in the cladding. The cross section of the incident X-ray beam is much greater than the core cross section at the entrance. It causes a significant amount of X-ray energy to be transmitted through the cladding, leading to unwanted background, and it also affects the far-field pattern. Consequently, the waveguide has to be

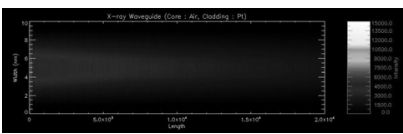

(a) $10 \mathrm{~nm}$

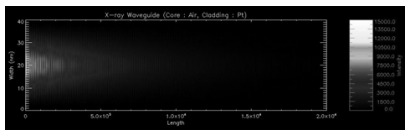

(c) $40 \mathrm{~nm}$

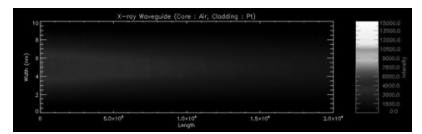

(b) $20 \mathrm{~nm}$

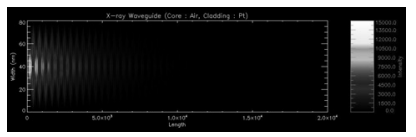

(d) $80 \mathrm{~nm}$
FIG. 2. The propagating modes in the air core waveguides with $\mathrm{Pt}$ cladding for different core thicknesses at an X-ray energy of $8.0 \mathrm{keV}$. 


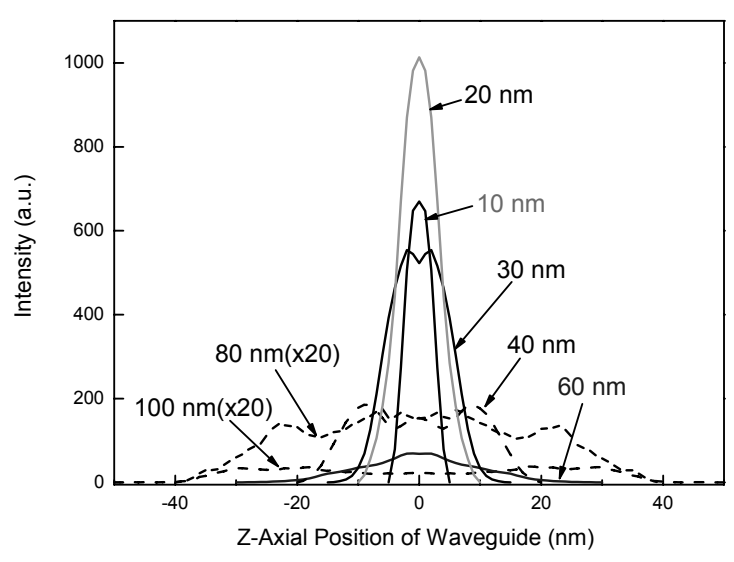

FIG. 3. The mode profiles in the Be core waveguides with $\mathrm{Pt}$ cladding at an energy of $8.0 \mathrm{keV}$.

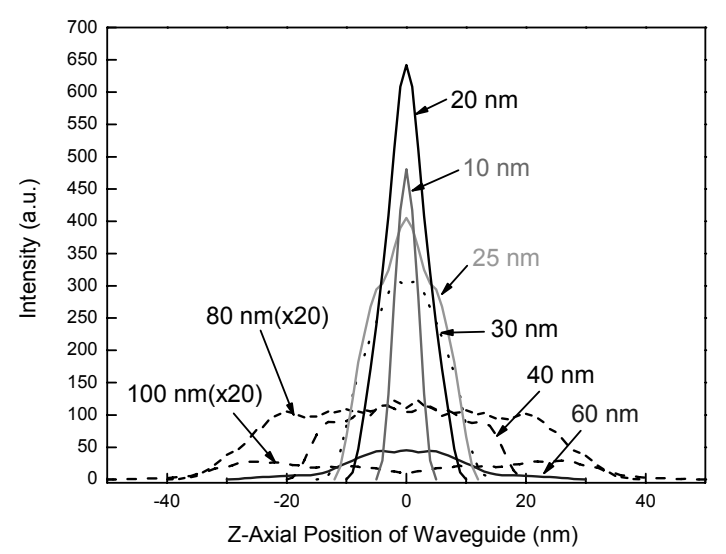

FIG. 4. The mode profiles in the air core waveguides with Pt cladding at an energy of $8.0 \mathrm{keV}$.

long enough to ensure that the ratio of transmission through the core and cladding is enough to achieve sufficient background reduction. The length of waveguide in our investigation is 2 millimeters, based on the absorption of the cladding materials [14]. In Fig. 2, the E-field intensities are shown for the air core waveguides with Pt cladding to find the dependence of the thickness of the core on the propagation modes. It is shown that a single mode generates in the core at a thickness of $20 \mathrm{~nm}$ see Fig. 2 (b). The single mode is clearly formed in a core of $10 \mathrm{~nm}$, Fig. 2 (a), but the intensity is about $40 \%$ lower than that for the core thickness of $20 \mathrm{~nm}$. Fig. 2 (c, d) show that multimodes form at a thickness of 40 , and $80 \mathrm{~nm}$ at an X-ray energy of $8.0 \mathrm{keV}$.

The mode profiles in the beryllium (Be) core waveguides with the platinum $(\mathrm{Pt})$ cladding are shown in Fig. 3. The single modes $\left(\mathrm{TE}_{0}\right)$ propagate with a core thickness of up to $20 \mathrm{~nm}$ see Fig. 3. The $\mathrm{TE}_{1}$ mode appears at a core thickness of $30 \mathrm{~nm}$ in Fig. 3. The $\mathrm{TE}_{2}$ mode is formed and the E-field intensity at the exit is dramatically reduced with a Be core thickness of $40 \mathrm{~nm}$. The multimode propagations are observed for

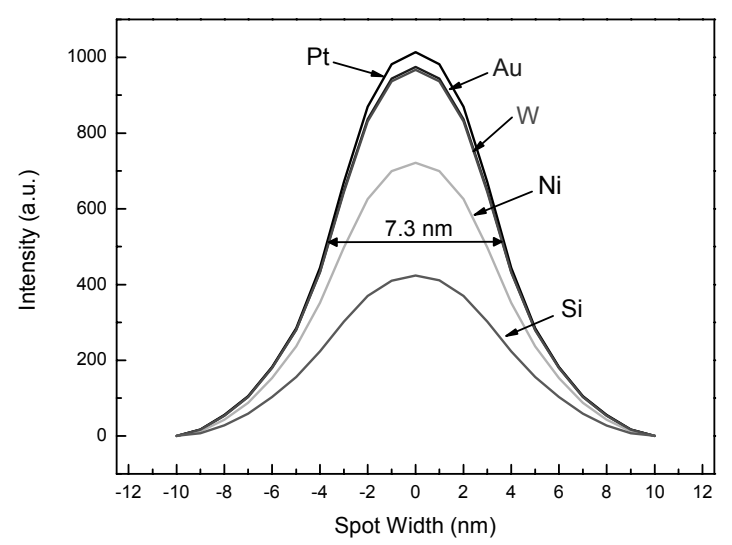

FIG. 5. The intensities at the exit of the Be core waveguides with a core thickness of $20 \mathrm{~nm}$ for different cladding materials.

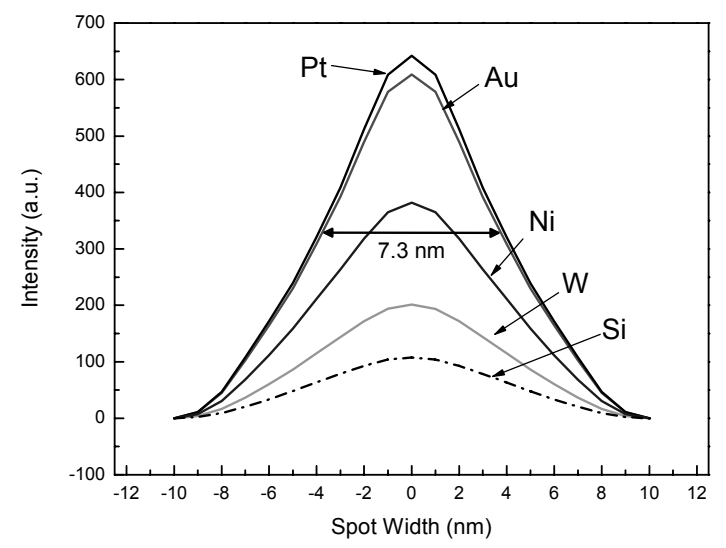

FIG. 6. The intensities of the air core waveguides with a core thickness of $20 \mathrm{~nm}$ for different cladding materials.

core thicknesses of $60 \mathrm{~nm}$ and above. The intensities from these waveguides reach $15 \%(60 \mathrm{~nm})$ and $0.2 \%$ $(100 \mathrm{~nm})$ of the single mode, respectively. The E-field intensities of the air core X-ray waveguides with the platinum cladding layers are shown in Fig. 4. The highest E-field intensity of this waveguide is $65 \%$ of the single mode, which radiates from the beryllium core waveguide in Fig. 3. The unexpected results may be caused by a large critical angle of the total reflection between the air-Pt interface. A much larger number of reflected rays at the interface of an air core and the cladding layer of the waveguide bounce back and forth within the core than in the Be core. So, more interference that acts to cancel each other out while traveling along the air core may occur in the air waveguide with $\mathrm{Pt}$ cladding. Thus the E-field intensity at the exit of the air channel waveguide is smaller than in the Be-cored one, even though the absorption of air is 100 times smaller than its Be equivalent. The trends of mode propagation in the air core waveguide are similar to the Be core waveguide, and are shown in Fig. 4.

The E-field intensities of the single mode at the exit 
TABLE 1. The number of modes and the thickness of the core for the air- $\mathrm{W}$ and the Be- $\mathrm{W}$ waveguides.

\begin{tabular}{c|c|c|c}
\hline \hline Type of waveguide & Number of mode & Thickness range [nm] & Max. thickness[nm] \\
\hline \multirow{3}{*}{ Be(core)-W(cladding) } & 1 & $8.5249 \leq \mathrm{d} \leq 17.049$ & \multirow{2}{*}{17.05} \\
\cline { 2 - 3 } & 2 & $17.049 \leq \mathrm{d} \leq 25.575$ & \\
\cline { 2 - 3 } & 3 & $25.575 \leq \mathrm{d} \leq 34.100$ & \multirow{2}{*}{1} \\
\cline { 2 - 3 } Air(core)-W(cladding) & 1 & $7.9836 \leq \mathrm{d} \leq 15.967$ & \multirow{2}{*}{15.97} \\
\cline { 2 - 3 } & 2 & $15.967 \leq \mathrm{d} \leq 23.951$ & \\
\cline { 2 - 3 } & 3 & $23.951 \leq \mathrm{d} \leq 31.934$ & \\
\hline
\end{tabular}

of the waveguide for different cladding materials are shown in Figs. 5-6. The $20 \mathrm{~nm}$ thick Be core waveguides with different claddings, i.e., $\mathrm{Pt}, \mathrm{Au}, \mathrm{W}, \mathrm{Ni}$, and $\mathrm{Si}$, are shown in Fig 5. The spot size of $7.3 \mathrm{~nm}$ in FWHM is obtained from the waveguides with $\mathrm{Pt}, \mathrm{Au}$ and $\mathrm{W}$ cladding layers. The intensities of the E-field also remain equivalent for those three cladding layers. The intensities for the single mode radiating from theair channel waveguides are shown in Fig. 6. In this case, the two waveguides with $\mathrm{Pt}$ and $\mathrm{Au}$ cladding reached about $65 \%$ of the Be core waveguide. The propagating mode intensity in the $\mathrm{W}$ cladding waveguide is shown to be far less than the intensity from the Be-W waveguide. The low reflectivity and high absorption of $\mathrm{W}$ may bring about the low intensity at the exit of the waveguide. Unfortunately, the Si cladding waveguides did not show good results either with an air or a Be core. In Table 1, the thickness of the core layers is determined for practical applications to fabricate the $\mathrm{X}$-ray waveguides of the $\mathrm{Be}-\mathrm{W}$ and the air- $\mathrm{W}$ system. A maximum thickness of $17.05 \mathrm{~nm}$ is obtained for the Be core waveguide with $\mathrm{W}$ cladding, and the maximum thickness of the air core waveguide with $\mathrm{W}$ cladding is $15.96 \mathrm{~nm}$.

\section{CONCLUSION}

Propagation of modes in the X-ray waveguides are investigated with numerical calculations based on parabolic wave equations. The E-field intensities in the core layer are calculated at an X-ray energy of $8.0 \mathrm{keV}$ for an air and a Be core withdifferent cladding layers such as $\mathrm{Pt}, \mathrm{Au}, \mathrm{W}, \mathrm{Ni}$ and $\mathrm{Si}$ to determine the dependence of materials. The highest E-field intensity at the exit is radiated at a thickness of $20 \mathrm{~nm}$ in the $\mathrm{Be}$ waveguide with the $\mathrm{Pt}$ cladding. However, the intensity from the waveguide with an air core- $\mathrm{Pt}$ cladding reaches $64 \%$ of the Be-Pt waveguide. The dependence on the thickness of the core, which is a major parameter for generating a single mode in the waveguide, is investigated for air-Pt, and the Be-Pt waveguides at an X-ray energy of $8.0 \mathrm{keV}$. The mode profiles at the exit show the single modes at up to a thickness of $20 \mathrm{~nm}$ for the air-Pt and the Be-Pt waveguides. The maximum thicknesses of the core are also determined for practical utilization of the waveguides using tungsten as a cladding material.

\section{ACKNOWLEDGMENT}

This work was supported by the Korea Science and Engineering Foundation (KOSEF) grant (2008-02012) funded by the Korean government (MOST).

\section{REFERENCES}

[1] G. G. Schroer, and B. Lengeler, "Focusing Hard X-ray to Nanometers Dimensions by Adiabatically Focusing Lenses," Phys. Rev. Lett., vol. 94, pp. 1-4, 2005.

[2] C. G. Schroer, O. Kurapova, J. Patommel, P. Boye, J. Feldkamp, and B. Lengeler, M. Burghammer, C. Riekel, L. Vincze, A. van der Hart, and M. Küchler, "Hard x-ray nanoprobe based on refractive x-ray lenses," Appl. Phys. Lett., vol. 87, pp. 124103-12410, 2005.

[3] A. G. Michette. S. J. Pfauntsch, A. Erko, A, and A. Svintsov," Nanometer Focusing of X-ray with Modified Reflection Zone Plates," Opt., vol. 245, pp. 349-253, 2005.

[4] Y. Suzuki, N. Kamijo, S. Tamura, K. Honda, A. Takeuchi, S. Yamamoto, H. Sugiyama, K. Ohsumi, and M. Ando, "Hard X-ray Micro-beam Experiment at the Tristan Main Ring Test Beam-line of the KEK," J. Synchrotron Radiat., vol. 4, pp. 60-63, 1997.

[5] Gung-Chian Yin, Yen-Fang Song, Mau-Tsu Tang, Fu-Rong Chen, Keng S. Liang, Frederick W. Duewer, Michael Feser, Wenbing Yun, and Han-Ping D. Shieh, "30 $\mathrm{nm}$ resolution $\mathrm{x}$-ray imaging at $8 \mathrm{keV}$ using third order diffraction of a zone plate lens objective in a transmission microscope," Appl. Phys. Lett., vol. 89, pp. 221-122, 2006.

[6] Weilun Chao, Bruce D. Harteneck, J. Alexander Liddle, Erik H. Anderson and David T. Attwood, "Soft X-ray microscopy at a spatial resolution better than $15 \mathrm{~nm}$," Nature, vol. 435, pp. 1210-1213, 2005.

[7] H. C. Kang, J. Maser, G. B. Stephenson, C. Liu, R. 
Conley, A. T. Macrander, and S. Vogt, "Nanometer Linear Focusing of Hard X Rays by a Multilayer Laue Lens," Phys. Rev. Lett., vol. 96, pp. 127401- 127404, 2006.

[8] F. Pfeiffer, C. David, J. F. van der Veen, C. Bergemann, "Nanometer focusing properties of Fresnel zone plates described by dynamical diffraction theory," Phys. Rev., vol. B73, pp. 245331-245340, 2006.

[9] J. Buschbeck,1,2 I. Lindemann,1,2 L. Schultz,1,2 and S. Fähler1, "Growth, structure, and texture of epitaxial Fe100-xPdx films deposited on $\mathrm{MgO}(100)$ at room temperature: An x-ray diffraction study," Phys. Rev., vol. B76, pp. 205421-205428, 2007.

[10] Christian G. Schroer, "Focusing hard x rays to nanometer dimensions using Fresnel zone plates,” Phys. Rev., B74, pp.033405-033408, 2006.

[11] D. H. Bilderback, S. A. Hottman, and D. J. Thiel, "Nanometer Spatial Resolution Achieved in Hard X-ray Imaging and Lave Diffraction Experiments." Science, vol. 263, pp. 201-203, 1994.

[12] Y. P. Feng, S.K. Sinha, E. E. Fullerton, G. Grubel, D. Abemathy, D. P. Siddons, and J. B. Hastings, "X-ray Fraunhofer Diffraction Patterns from a Thin-Film Waveguide," Appl. phys. Lett., vol. 67, pp. 3647-3649, 1995.
[13] F. Pfeitter, T. Salditt, P. Hoghoj, I. Anderson, and N. Schell, "X-ray Waveguides with Multiple Guiding Layers,” Phys. Rev., vol. B62, pp. 16939-16943, 2000.

[14] C. Brgemann, H. Keymeulen, and J. F. Van der Veen, "Focusing X-ray Beams to Nanometer Dimensions," Phys. Rev. Lett., vol. 91, pp. 204801-204805, 2003.

[15] F. Pfeiffer, C. David, M. Burghammer, C. Rickel, and T. Salditt, "Two-Dimensional X-ray Waveguides and Point Sources,” Science, vol. 297, pp. 230-234, 2002.

[16] Jianwei Miao, Pambos Charalambous, Janos Kirz and David Sayre, "Extending the methodology of X-ray crystallography to allow imaging of micrometre- sized non-crystalline specimens," Nature, vol. 400, pp. 342344, 1999.

[17] J. Wang, M. J. Beyk and M. Caffrey, " ResonanceEnhanced X-rays in Thin-films," Science, vol. 258, pp. 775-778, 1992.

[18] M. J. Bedzyk, G. M. Bommarito and J. S. Schildkraut "X-ray Standing Waves at a Reflecting Mirror Surface," phys. Rev. Lett., vol. 62, pp. 1376-1379, 1989.

[19] Christian Fuhse, X-ray Waveguides and Waveguidebased Lensless Imaging, (Dissertation, Georg-August University, Germany, 2006.)

[20] J. W. Thomas, Numerical Partial Differential Equations, (Springer-Verlag, New York, 1997.) 